\title{
Optimization of Doping of Heterostructure during Manufacturing of P-I-N-Diodes
}

\author{
E.L. Pankratov ${ }^{1, *}$, E.A. Bulaeva ${ }^{2}$ \\ ${ }^{1}$ Nizhny Novgorod State University, 23 Gagarin Avenue, Nizhny Novgorod, 603950, Russia \\ ${ }^{2}$ Nizhny Novgorod State University of Architecture and Civil Engineering, 65 Il'insky Street, Nizhny Novgorod, 603950, Russia \\ *Corresponding Author: elp2004@mail.ru
}

Copyright $@ 2013$ Horizon Research Publishing All rights reserved.

\begin{abstract}
In this paper we introduce an approach to manufacture p-i-n-diodes with higher compactness. The introduced approach based on implantation of ions of dopants in a semiconductor heterostructure and optimization of annealing.
\end{abstract}

Keywords P-I-N-Diodes, Optimization of Manufacturing, Analytical Approach for Modeling

\section{Introduction}

During last decades one can find intensive development of elements of integrated circuits ( $p$ - $n$-junctions, field-effect and bipolar transistors, et al) and their discrete analogs ${ }^{[1-7]}$. One way of the development is increasing of sharpness of $p$ - $n$-junctions and homogeneity of distributions of dopants in doped areas. The increasing of sharpness leads to decrease switching time of the $p$ - $n$-junctions during their operating. At the same time increasing of homogeneity of dopant distribution in a $p$ - $n$-junction gives us possibility to increase current density in the devices or to decrease dimensions of the devices at fixed value of heating during operating.

To increase sharpness of $p$ - $n$-junctions it could be used laser and microwave types of annealing ${ }^{[8-10]}$, availability of defects in doped structure (for example, availability of defects due to radiation processing) ${ }^{[11]}$, inhomogeneity of heterostructure ${ }^{[12-14]}$. In this paper based on recently introduced approach ${ }^{[12-14]}$ we consider an approach to decrease dimensions of a $p$ - $i$-n-diode due to increasing of homogeneity of distributions of dopants in doped areas and increasing of speed of decreasing of concentrations of the dopants near interfaces between doped and undoped areas. To solve this problem we consider a heterostructure, which consist of a substrate and two epitaxial layers (see Fig. 1). A dopant has been implanted in the substrate before growth the first epitaxial layers. The dopant produced necessary type of conductivity in the substrate $(n$ or $p$ ). Farther an undoped epitaxial layer has been grown. The second epitaxial layer has been grown after the first one. The second type of dopant has been implanted into the second epitaxial layer to produce reverse type of conductivity ( $p$ or $n$ ) into the layer. Father we consider annealing of radiation defects. Quantity of the defects decreases during the annealing. As an accompanying result of the annealing one can obtain spreading of implanted dopant distributions in the substrate and in the second epitaxial layer until interfaces of the layers of heterostructure. Main aim of the present paper is determination of conditions to manufacture more compact $p$ - $i$ - $n$-diode.

\section{Method of Solution}

To solve our aim we determine spatiotemporal distributions of concentrations of dopants and radiation defects. We determine the distributions by solving the second Fick's low ${ }^{[1,15-17]}$.

$$
\frac{\partial C(x, t)}{\partial t}=\frac{\partial}{\partial x}\left[D_{C} \frac{\partial C(x, t)}{\partial x}\right]
$$




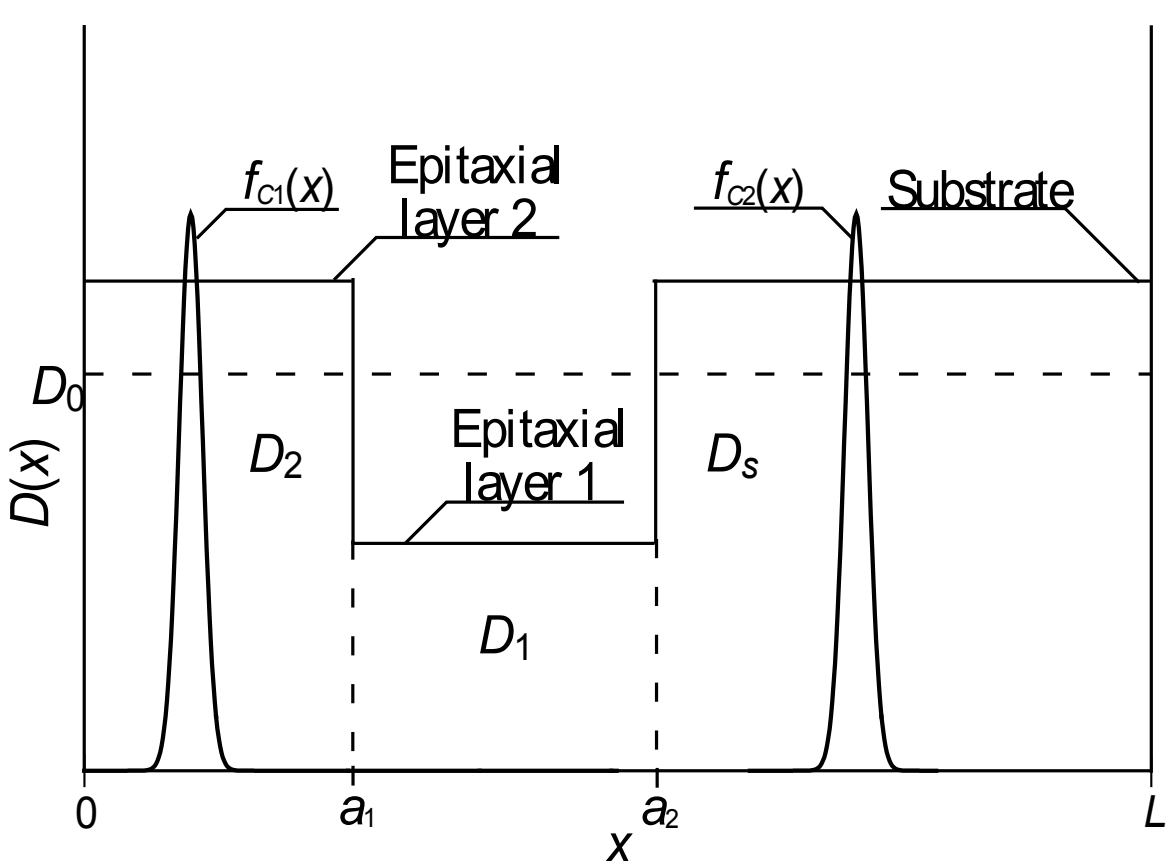

Figure 1. Heterostructure with three layers and initial (before annealing) initial distributions of dopants. Here $D_{s}-$ is the dopant diffusion coefficient in the substrate; $D_{1}$ and $D_{2}$ - are dopant diffusion coefficients in the epitaxial layers; $D_{0}$ - is the average value of dopant diffusion coefficient in the heterostructure

$$
\begin{aligned}
& \left\{\begin{array}{l}
\frac{\partial I(x, t)}{\partial t}=\frac{\partial}{\partial x}\left[D_{I}(x, T) \frac{\partial I(x, t)}{\partial x}\right]-k_{I, V}(x, T) I(x, t) V(x, t)-k_{I, I}(x, T) I^{2}(x, t) \\
\frac{\partial V(x, t)}{\partial t}=\frac{\partial}{\partial x}\left[D_{V}(x, T) \frac{\partial V(x, t)}{\partial x}\right]-k_{I, V}(x, T) I(x, t) V(x, t)-k_{V, V}(x, T) V^{2}(x, t)
\end{array}\right. \\
& \left\{\begin{array}{l}
\frac{\partial \Phi_{I}(x, t)}{\partial t}=\frac{\partial}{\partial x}\left[D_{\Phi I}(x, T) \frac{\partial \Phi_{I}(x, t)}{\partial x}\right]+k_{I, I}(x, T) I^{2}(x, t)-k_{I}(x, T) I(x, t) \\
\frac{\partial \Phi_{V}(x, t)}{\partial t}=\frac{\partial}{\partial x}\left[D_{\Phi V}(x, T) \frac{\partial \Phi_{V}(x, t)}{\partial x}\right]+k_{V, V}(x, T) V^{2}(x, t)-k_{V}(x, T) V(x, t)
\end{array}\right.
\end{aligned}
$$

With boundary and initial conditions

$$
\begin{gathered}
\left.\frac{\partial C(x, t)}{\partial x}\right|_{x=0}=0,\left.\frac{\partial C(x, t)}{\partial x}\right|_{x=L}=0,\left.\frac{\partial I(x, t)}{\partial x}\right|_{x=0}=0,\left.\frac{\partial I(x, t)}{\partial x}\right|_{x=L}=0,\left.\frac{\partial V(x, t)}{\partial x}\right|_{x=0}=0, \\
\left.\frac{\partial V(x, t)}{\partial x}\right|_{x=L}=0,\left.\frac{\partial \Phi_{I}(x, t)}{\partial x}\right|_{x=0}=0,\left.\frac{\partial \Phi_{I}(x, t)}{\partial x}\right|_{x=L}=0,\left.\frac{\partial \Phi_{V}(x, t)}{\partial x}\right|_{x=0}=0,\left.\frac{\partial \Phi_{V}(x, t)}{\partial x}\right|_{x=L}=0, \\
C(x, 0)=f_{C}(x), I(x, 0)=f_{I}(x), V(x, 0)=f_{V}(x), \Phi_{I}(x, 0)=f_{\Phi I}(x), \Phi_{V}(x, 0)=f_{\Phi V}(x) .(4)
\end{gathered}
$$

Here $\mathrm{C}(\mathrm{x}, \mathrm{t}), \mathrm{I}(\mathrm{x}, \mathrm{t}), \mathrm{V}(\mathrm{x}, \mathrm{t}), \Phi_{\mathrm{I}}(\mathrm{x}, \mathrm{t}), \Phi_{\mathrm{V}}(\mathrm{x}, \mathrm{t})$ are spatiotemporal distributions of concentrations of dopants, point radiation defects (interstitials and vacancies) and simplest complexes of point radiation defects (diinterstitials and divacancies); $\mathrm{D}_{\mathrm{C}}, \mathrm{D}_{\mathrm{I}}$, $\mathrm{D}_{\mathrm{V}}, \mathrm{D}_{\Phi \mathrm{I}}$ and $\mathrm{D}_{\Phi \mathrm{V}}$ are diffusion coefficients of dopants and radiation defects (index of diffusion coefficients shows their appurtenance); $\mathrm{k}_{\mathrm{I}, \mathrm{I}}(\mathrm{x}, \mathrm{T})$ and $\mathrm{k}_{\mathrm{V}, \mathrm{V}}(\mathrm{x}, \mathrm{T})$ are parameters of generation of simplest complexes of radiation defects; $\mathrm{k}_{\mathrm{I}}(\mathrm{x}, \mathrm{T})$ and $\mathrm{k}_{\mathrm{V}}(\mathrm{x}, \mathrm{T})$ are parameters of decay of simplest complexes of radiation defects; $\mathrm{k}_{\mathrm{I}, \mathrm{V}}(\mathrm{x}, \mathrm{T})$ is the parameter of recombination of radiation defects; $\mathrm{T}$ is the temperature of annealing. The first term in the right side of the Eq.(2) corresponds to diffusion of point radiation defects, the second term corresponds to recombination of point radiation defects, the third term corresponds to 
generation of simplest complexes of point radiation defects. Value of dopant diffusion coefficients depends on properties of materials in considered heterostructure (Fig. 1), speed of heating and cooling of the heterostructure (framework Arrhenius low). Redistribution of dopant also depends on level of doping of materials and defects. Dependences of dopant diffusion coefficient on parameters could be approximated by the following relation [15]

$$
D_{C}=D_{L}(x, T)\left[1+\varsigma_{1} \frac{V(x, t)}{V^{*}}+\varsigma_{2} \frac{V^{2}(x, t)}{\left(V^{*}\right)^{2}}\right]\left[1+\xi \frac{C^{\gamma}(x, t)}{P^{\gamma}(x, T)}\right] .
$$

Here $D_{L}(x, T)$ is the spatial (due to inhomogeneity of heterostructure) and temperature (due to Arrhenius low) dependences of dopant diffusion coefficient; $P(x, T)$ is the limit of solubility of dopant; $V^{*}$ is the equilibrium distribution of vacancies; parameter $\gamma$ depends on properties of materials and could be integer in the interval $\gamma \in[1,3]$ [15]. Dependence of dopant diffusion coefficient on dopant concentration had been discussed in details in ${ }^{[15]}$.

Spatiotemporal distribution of concentrations of dopant and radiation defects we determine by method of averaging of function corrections ${ }^{[18]}$ with decreased quantity of iterative steps ${ }^{[19]}$. Framework the approach we consider distributions of concentrations of dopant and radiation defects with averaged diffusion coefficients $D_{0 L}, D_{0 I}, D_{0 V}, D_{0 \Phi I}$, $D_{0 \Phi V}$, zero values of parameters of recombination of point radiation defects, zero values of parameters of generation and decay of complexes of radiation defects as initial-order approximations of the above concentrations. The initial-order approximations of concentrations of dopant and radiation defects could be written as

$$
\begin{array}{r}
C_{1}(x, t)=\frac{2 \pi}{L} \sum_{n=1}^{\infty} F_{n C} c_{n}(x) e_{n C}(t), \quad I_{1}(x, t)=\frac{2 \pi}{L} \sum_{n=1}^{\infty} F_{n I} c_{n}(x) e_{n I}(t), \quad V_{1}(x, t)=\frac{2 \pi}{L} \times \\
\times \sum_{n=1}^{\infty} F_{n V} c_{n}(x) e_{n V}(t), \quad \Phi_{I 1}(x, t)=\frac{2 \pi}{L} \sum_{n=1}^{\infty} F_{n} \Phi_{I} c_{n}(x) e_{n} \Phi_{I}(t), \quad \Phi_{V 1}(x, t)=\frac{2 \pi}{L} \sum_{n=1}^{\infty} F_{n \Phi_{V}} c_{n}(x) e_{n \Phi_{V}}(t),
\end{array}
$$

where $F_{n \rho}=\int_{0}^{L} c_{n}(u) f_{\rho}(u) d u ; e_{n \rho}(x)=\exp \left(-\pi^{2} n^{2} D_{0 \rho} / L^{2}\right) ; c_{n}(x)=\cos (\pi n x / L)$.

We determine approximations of concentrations with the second and higher orders by using standard iterative procedure of method of averaging of function corrections ${ }^{[18]}$. Framework the procedure to determine $n$-order approximations of concentrations of dopant and radiation defects we shall replace the concentrations $C(x, t), I(x, t), V(x, t), \Phi_{I}(x, t)$ and $\Phi_{V}(x, t)$ in the right sides of Eqs. (1)-(3) on sum of average value of the $n$-order approximation and approximation with the previous order, i.e. $\alpha_{n \rho}+\rho_{n-1}(x, t)$. The replacement gives us possibility to obtain equations for the second-order approximations of the above concentrations in the following form

$$
\begin{aligned}
& \frac{\partial C_{2}(x, t)}{\partial t}=\frac{\partial}{\partial x}\left(D_{L}(x, T)\left[1+\varsigma_{1} \frac{V(x, t)}{V^{*}}+\varsigma_{2} \frac{V^{2}(x, t)}{\left(V^{*}\right)^{2}}\right]\left\{1+\xi \frac{\left[\alpha_{2 C}+C_{1}(x, t)\right]^{\gamma}}{P^{\gamma}(x, T)}\right\} \frac{\partial C_{1}(x, t)}{\partial x}\right)(6) \\
& \left\{\begin{array}{l}
\frac{\partial I_{2}(x, t)}{\partial t}=\frac{\partial}{\partial x}\left[D_{I}(x, T) \frac{\partial I_{1}(x, t)}{\partial x}\right]- \\
-k_{I, V}(x, T)\left[\alpha_{2 I}+I_{1}(x, t)\right]\left[\alpha_{2 V}+V_{1}(x, t)\right]-k_{I, I}(x, T)\left[\alpha_{2 I}+I_{1}(x, t)\right]^{2} \\
\frac{\partial V_{2}(x, t)}{\partial t}=\frac{\partial}{\partial x}\left[D_{V}(x, T) \frac{\partial V_{1}(x, t)}{\partial x}\right]- \\
-k_{I, V}(x, T)\left[\alpha_{2 I}+I_{1}(x, t)\right]\left[\alpha_{2 V}+V_{1}(x, t)\right]-k_{V, V}(x, T)\left[\alpha_{2 V}+V_{1}(x, t)\right]^{2}
\end{array}\right.
\end{aligned}
$$




$$
\left\{\begin{array}{l}
\frac{\partial \Phi_{I 2}(x, t)}{\partial t}=\frac{\partial}{\partial x}\left[D_{\Phi I}(x, T) \frac{\partial \Phi_{I 1}(x, t)}{\partial x}\right]+k_{I, I}(x, T) I^{2}(x, t)-k_{I}(x, T) I(x, t) \\
\frac{\partial \Phi_{V 2}(x, t)}{\partial t}=\frac{\partial}{\partial x}\left[D_{\Phi V}(x, T) \frac{\partial \Phi_{V 1}(x, t)}{\partial x}\right]+k_{V, V}(x, T) V^{2}(x, t)-k_{V}(x, T) V(x, t)
\end{array}\right.
$$

Integration on time of the left and right sides of Eqs. (6)-(8) gives us possibility to obtain the second-order approximations of concentrations of dopant and radiation defects in the following form

$$
\begin{aligned}
& C_{2}(x, t)=\frac{\partial}{\partial x}\left(\int_{0}^{t} D_{L}(x, T)\left[1+\varsigma_{1} \frac{V(x, \tau)}{V^{*}}+\varsigma_{2} \frac{V^{2}(x, \tau)}{\left(V^{*}\right)^{2}}\right] \times\right. \\
& \left.\times\left\{1+\xi \frac{\left[\alpha_{2 C}+C_{1}(x, \tau)\right]^{\gamma}}{P^{\gamma}(x, T)}\right\} \frac{\partial C_{1}(x, \tau)}{\partial x} d \tau\right)+f_{C}(x) \\
& I_{2}(x, t)=\frac{\partial}{\partial x}\left[\int_{0}^{t} D_{I}(x, T) \frac{\partial I_{1}(x, \tau)}{\partial x} d \tau\right]-\int_{0}^{t} k_{I, I}(x, T)\left[\alpha_{2 I}+I_{1}(x, \tau)\right]^{2} d \tau- \\
& -\int_{0}^{t} k_{I, V}(x, T)\left[\alpha_{2 I}+I_{1}(x, \tau)\right]\left[\alpha_{2 V}+V_{1}(x, \tau)\right] d \tau+f_{I}(x) \\
& V_{2}(x, t)=\frac{\partial}{\partial x}\left[\int_{0}^{t} D_{V}(x, T) \frac{\partial V_{1}(x, \tau)}{\partial x} d \tau\right]-\int_{0}^{t} k_{V, V}(x, T)\left[\alpha_{2 V}+V_{1}(x, \tau)\right]^{2} d \tau- \\
& -\int_{0}^{t} k_{I, V}(x, T)\left[\alpha_{2 I}+I_{1}(x, \tau)\right]\left[\alpha_{2 V}+V_{1}(x, \tau)\right] d \tau+f_{V}(x) \\
& \left\{\begin{aligned}
\Phi_{I 2}(x, t)=\frac{\partial}{\partial x}\left[\int_{0}^{t} D_{\Phi I}(x, T) \frac{\partial \Phi_{I 1}(x, \tau)}{\partial x} d \tau\right]+ & \int_{0}^{t} k_{I, I}(x, T) I^{2}(x, \tau) d \tau- \\
& -\int_{0}^{t} k_{I}(x, T) I(x, \tau) d \tau+f_{\Phi I}(x) \\
\Phi_{V 2}(x, t)=\frac{\partial}{\partial x}\left[\int_{0}^{t} D_{\Phi V}(x, T) \frac{\partial \Phi_{V 1}(x, \tau)}{\partial x} d \tau\right]+ & \int_{0}^{t} k_{V, V}(x, T) V^{2}(x, \tau) d \tau- \\
& -\int_{0}^{t} k_{V}(x, T) V(x, \tau) d \tau+f_{\Phi V}(x)
\end{aligned}\right.
\end{aligned}
$$

We determine average values of the second-order approximations of desired concentrations by the standard relation ${ }^{[18]}$

$$
\alpha_{2 \rho}=\frac{1}{\Theta L} \int_{0}^{\Theta L} \int_{0}^{L}\left[\rho_{2}(x, t)-\rho_{1}(x, t)\right] d x d t
$$

Substitution of the relations (6a)-(8a) in the relation (9) gives us possibility to obtain the desired average values $\alpha_{2 \rho}$

$$
\alpha_{2 C}=\frac{1}{L} \int_{0}^{L} f_{C}(x) d x
$$




$$
\left\{\begin{array}{l}
\alpha_{2 I}=\left\{\left(1+A_{I V 01}+A_{I I 10}+\alpha_{2 V} A_{I V 00}\right)^{2}-4\left[\alpha_{2 V} A_{I V 10}-A_{I I 20}+A_{I V 11}-\frac{1}{L} \int_{0}^{L} f_{I}(x) d x\right] \times\right. \\
\left.\times A_{I I 00}\right\}^{\frac{1}{2}} \frac{1}{2 A_{I I 00}}-\frac{1+A_{I V 01}+A_{I I 10}+\alpha_{2 V} A_{I V 00}}{2 A_{I I 00}} \\
\alpha_{2 V}=\frac{1}{2 B_{4}} \sqrt{\frac{\left(B_{3}+A\right)^{2}}{4}-4 B_{4}\left(y+\frac{B_{3} y-B_{1}}{A}\right)}-\frac{B_{3}+A}{4 B_{4}}
\end{array}\right.
$$

where $A_{a b i j}=\frac{1}{\Theta L} \int_{0}^{\Theta}(\Theta-t) \int_{0}^{L} k_{a, b}(x, T) I_{1}^{i}(x, t) V_{1}^{j}(x, t) d x d t, B_{4}=A_{I V 00}^{2} A_{I V 00}^{2}-2\left(A_{I V 00}^{2}-A_{I I 00} \times\right.$ $\left.\times A_{V V 00}\right)^{2}, B_{3}=A_{I V 00} A_{I V 00}^{2}+A_{I V 01} A_{I V 00}^{3}+A_{I V 00} A_{I I 10} A_{I V 00}^{2}-4 A_{I V 10} A_{I I 00} A_{I V 00}^{2} 2 A_{I V 00} A_{I V 01} A_{I V 00}^{2}-$ $-4\left(A_{I V 00}^{2}-A_{I I 00} A_{V V 00}\right)\left[2 A_{I V 01} A_{I V 00}+2 A_{I V 00}\left(1+A_{I V 01}+A_{I I 10}\right)-2 A_{I I 00}\left(A_{I V 10}+A_{V V 10}+1\right)\right], B_{2}=$ $=A_{I V 00}^{2}\left\{\left(1+A_{I V 01}+A_{I I 10}\right)^{2}+A_{I V 00}^{2} A_{I V 01}^{2}-4 A_{I I 00}\left[A_{I V 11}-A_{I I 20}-\frac{1}{L} \int_{0}^{L} f_{I}(x) d x\right]+A_{I V 00} A_{I V 01} \times\right.$ $\left.\times 2\left(A_{I V 00}+A_{I V 00} A_{I V 01}+A_{I V 00} A_{I I 10}-4 A_{I V 10} A_{I I 00}\right)\right\}\left\{\left[2 A_{I V 00}\left(1+A_{I V 01}+A_{I I 10}\right)+2 A_{I V 01} A_{I V 00}-\right.\right.$ $\left.-2 A_{I I 00}\left(A_{I V 10}+A_{V V 10}+1\right)\right]^{2}+2\left[A_{I V 01}\left(1+A_{I V 01}+A_{I I 10}\right)+\frac{2}{L} A_{I I 00} \int_{0}^{L} f_{V}(x) d x-2 A_{I I 00}\left(A_{V V 20}-\right.\right.$ $\left.\left.-A_{I V 11}\right)+A_{I V 01}\left(1+A_{I V 01}+A_{I I 10}\right)\right]\left[2 A_{I V 00}\left(1+A_{I V 01}+A_{I I 10}\right)-2 A_{I I 00}\left(A_{I V 10}+A_{V V 10}+1\right)+A_{I V 01} \times\right.$ $\left.\left.\times 2 A_{I V 00}\right]\right\}, \quad B_{1}=2 A_{I V 00} A_{I V 01}\left(1+A_{I V 01}+A_{I I 10}\right)^{2}-8 A_{I V 00} A_{I V 01} A_{I I 00}\left[A_{I V 11}-\frac{1}{L} \int_{0}^{L} f_{I}(x) d x-A_{I I 20}\right]+$ $+A_{I V 01}^{2}\left(A_{I V 00}+A_{I V 00} A_{I V 01}+A_{I V 00} A_{I I 10}-4 A_{I V 10} A_{I I 00}\right)-2\left[A_{I V 01}\left(1+A_{I V 01}+A_{I I 10}\right)-2 A_{I I 00} \times\right.$ $\left.\times\left(A_{V V 20}-A_{I V 11}\right)+\frac{2}{L} A_{I I 00} \int_{0}^{L} f_{V}(x) d x+A_{I V 01}\left(1+A_{I V 01}+A_{I I 10}\right)\right]\left[2 A_{I V 00}\left(1+A_{I V 01}+A_{I I 10}\right)+\right.$ $\left.+2 A_{I V 01} A_{I V 00}-2 A_{I I 00}\left(A_{I V 10}+A_{V V 10}+1\right)\right], B_{0}=4 A_{I I 00} A_{I V 01}^{2}\left[A_{I I 20}+\frac{1}{L} \int_{0}^{L} f_{I}(x) d x-A_{I V 11}\right]+$ $+A_{I V 01}^{2}\left(A_{I V 01}+A_{I I 10}+1\right)^{2}-\left[A_{I V 01}\left(1+A_{I V 01}+A_{I I 10}\right)-2 A_{I I 00}\left(A_{V V 20}-A_{I V 11}\right)+A_{I V 01}\left(A_{I V 01}+\right.\right.$ $\left.\left.+1+A_{I I 10}\right)+\frac{2}{L} A_{I I 00} \int_{0}^{L} f_{V}(x) d x\right]^{2}, A=\sqrt{8 y+B_{3}^{2}-4 B_{2}}, y=\sqrt[3]{\sqrt{q^{2}+p^{3}}-q}+\frac{B_{2}}{6}-$ $-\sqrt[3]{\sqrt{q^{2}+p^{3}}+q}, \quad p=\left[3\left(B_{1} B_{3}-4 B_{0}\right)-B_{2}^{2}\right] / 36, q=\frac{B_{2}^{3}}{216}+\frac{B_{0}\left(4 B_{2}-B_{3}^{2}\right)-B_{1}^{2}}{8}+\frac{B_{2}}{24} \times$ $\times\left(B_{1} B_{3}-4 B_{0}\right)$ 


$$
\left\{\begin{array}{l}
\alpha_{2 \Phi_{I}}=A_{I I 20}-\frac{1}{\Theta L} \int_{0}^{\Theta}(\Theta-t) \int_{0}^{L} k_{I}(x, T) I(x, t) d x d t+\frac{1}{L} \int_{0}^{L} f_{\Phi I}(x) d x \\
\alpha_{2 \Phi_{V}}=A_{V V 20}-\frac{1}{\Theta L} \int_{0}^{\Theta}(\Theta-t) \int_{0}^{L} k_{V}(x, T) V(x, t) d x d t+\frac{1}{L} \int_{0}^{L} f_{\Phi V}(x) d x
\end{array} .\right.
$$

Analysis of spatiotemporal distributions of concentrations of dopant and radiation defects has been done analytically by using the second-order approximations of concentrations framework the method of averaging of function correction with decrease quantity of iteration steps. The second-order approximation is usually enough good approximation to obtain qualitative results and some quantitative estimations. Results of analytical calculations have been checked by comparison of the results with results of numerical simulation.

\section{Discussion}

In this section we analyzed dynamics of redistribution of dopant and radiation defects during annealing. Some typical distributions of dopant for different values of difference between dopant diffusion coefficients are presented in Fig. 2 . The figure shows, that interface between layers of heterostructure gives us possibility to increase homogeneity of dopant distributions and at the same time to increase speed of decreasing of dopant concentration near interface. In this situation some parameters of technological process (such as energy of implanted ions, thicknesses and materials of layers of heterostructure) could not be selected independently. One can obtain spreading of dopant distributions during annealing. The parameters should be choose so, that distributions of dopants during their spreading should achieve to interfaces between layers of heterostructure. If dopanst did not achieve nearest interfaces, one shall use additional annealing of dopant. To optimize the additional annealing time we used recently introduce criterion ${ }^{[12-14]}$. Framework the criterion we determine real distribution of dopant by step-wise distribution (curve 1 of Fig. 3). Optimal values of additional annealing time have been calculated by minimization the following mean-squared error

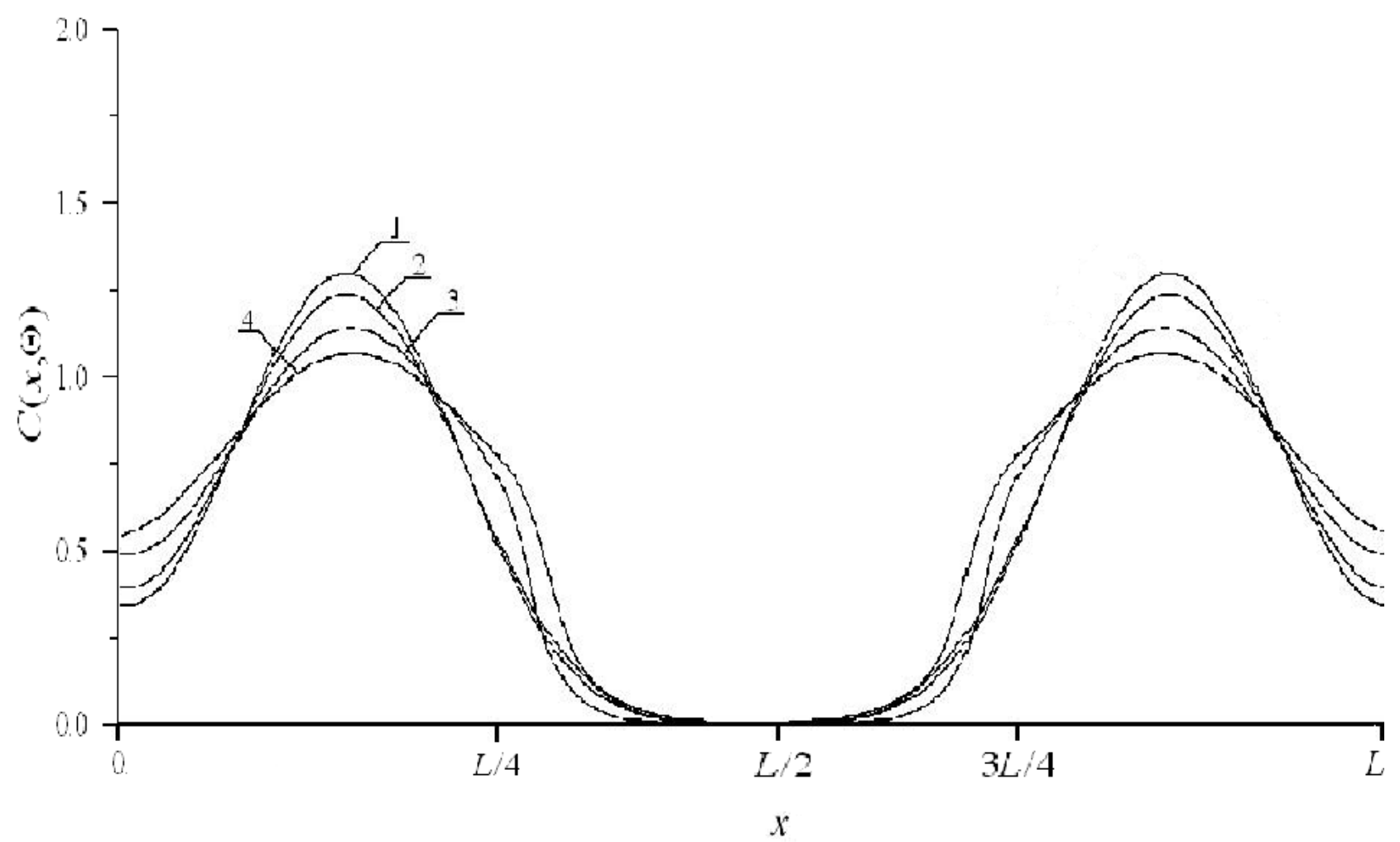

Figure 2. Spatial distributions of dopants after annealing with continuance $\Theta=0,0045 L^{2} / D_{0}$ (curves 1,3 ) and $\Theta=0,0057 L^{2} / D_{0}$ (curves 2,4 ). Curves 1 and 2 are calculated distributions in a homogenous sample. Curves 3 and 4 are calculated distributions in a heterostructure for $D_{2}>D_{1}$ and $D_{s}>D_{1}$. Interfaces between layers of heterostructure have coordinates $a_{1}=L / 4$ and $a_{2}=3 L / 4$ 


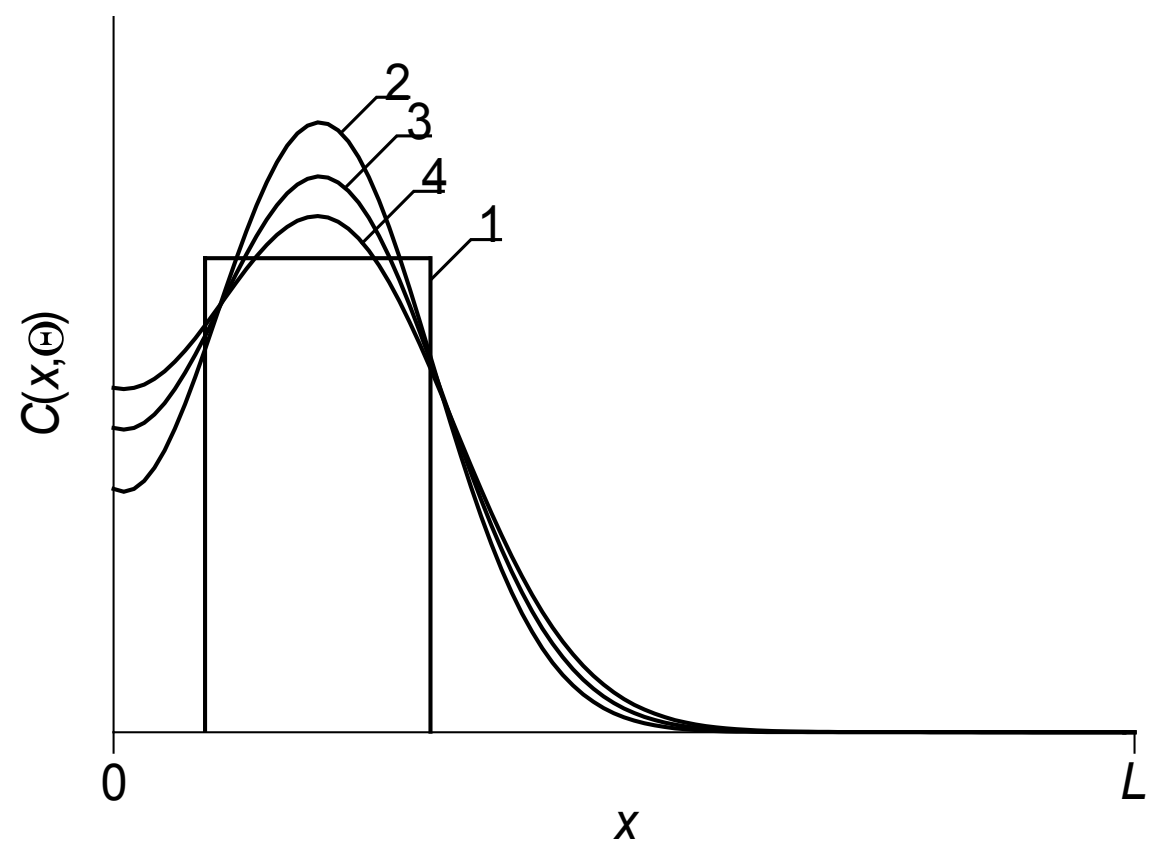

Figure 3. Spatial distributions of infused dopant in heterostructure. Curve 1 is required idealized distribution of dopant. Curves 2-4 are real distributions of infused dopant in heterostructure for different values of annealing time (increasing of number of curve corresponds to increasing of annealing time)

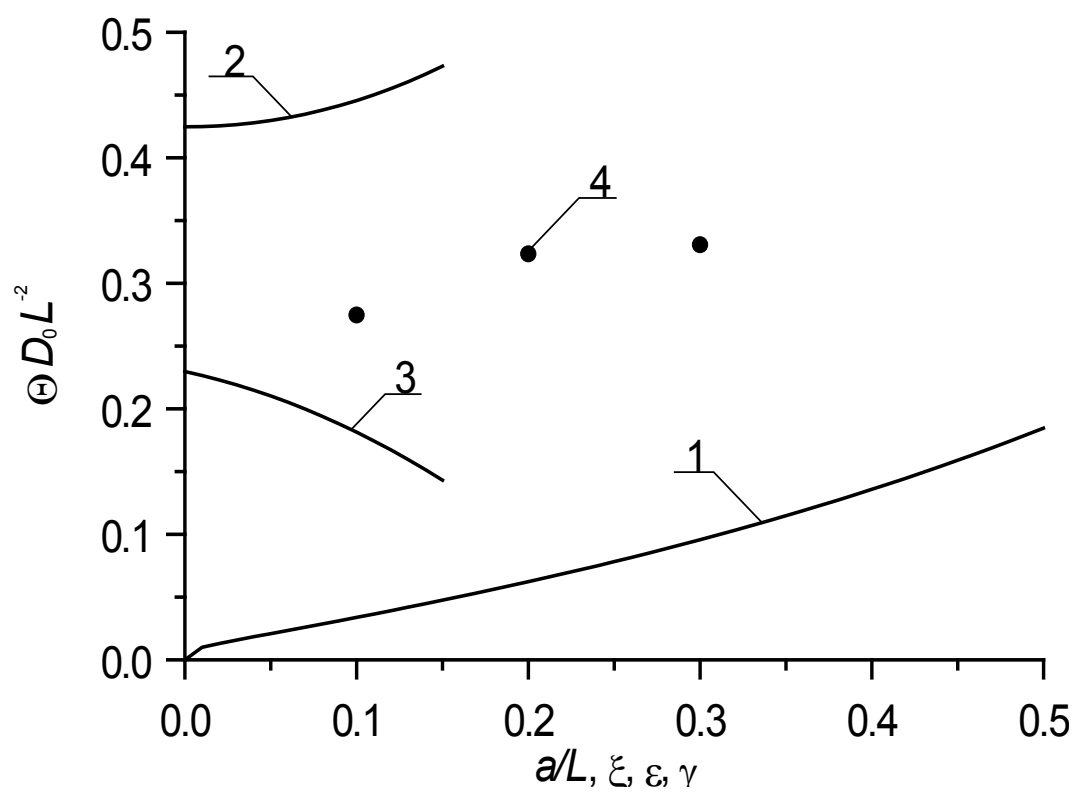

Figure 4. Dependences of dimensionless optimal time of additional annealing, which have been calculated by minimization the mean squared error Eq. (6) on several parameters. Curve 1 is the dependence of optimal annealing time on the ratio $a_{1} / L_{x}$ (dependence of optimal annealing time on the ratio $a_{2} / L_{x}$ is the similar to the dependence on $a_{1} / L$ ) for pairwise equality of dopant diffusion coefficients and $\xi=\gamma=0$. Curve 2 is the dependence of optimal annealing time on the relation $\left(D_{1} / D_{S}-1\right)$ for $a_{1} / L=1 / 2$ (dependence of optimal annealing time on the ratio $\left(D_{2} / D_{S}-1\right)$ is the similar to the depen-dence on $\left(D_{1} / D_{S}-1\right)$ ), $\xi=\gamma=0$. Curve 3 is the dependence of optimal annealing time on the parameter $\xi$ for pairwise equality of dopant diffusion coefficients and $a_{1} / L=1 / 2, \gamma=0$. Curve 4 is the dependence of optimal annealing time on the parameter $\gamma$ for pairwise equality of dopant diffusion coefficients and $a_{1} / L=1 / 2, \xi=0$

$$
U=\frac{1}{L} \int_{0}^{L}[C(x, \Theta)-\psi(x)] d x
$$

where $\psi(x)$ is the approximation function from Fig. 3 (curve 1). Dependences of optimal annealing time on several parameters are presented on curve 4. 


\section{Conclusion}

In this paper we introduce an approach to manufacture more compact $p$ - $i$ - $n$-diodes due to implantation of ions of dopants in a semiconductor heterostructure and optimization of annealing. We also introduce an approach to model redistribution of dopants and radiation defects in the heterostructure, which gives us possibility to dispense without "joining" of solutions on interfaces between layers of the structure.

This work is supported by the contract 11.G34.31.0066 of the Russian Federation Government and educational fellowship for scientific research.

\section{REFERENCES}

[1] A. Kerentsev, V. Lanin, Power Electronics, Issue 1. P. 34 (2008).

[2] A.O. Ageev, A.E. Belyaev, N.S. Boltovets, V.N. Ivanov, R.V. Konakova, Ya.Ya. Kudrik, P.M. Litvin, V.V. Milenin, A.V. Sachenko. Semiconductors. Vol. 43 (7). P. 897-903 (2009).

[3] Jung-Hui Tsai, Shao-Yen Chiu, Wen-Shiung Lour, Der-Feng Guo. Semiconductors. Vol. 43 (7). P. 971-974 (2009).

[4] E.I. Goldman, N.F. Kukharskaya, V.G. Naryshkina, G.V. Chucheva. Semiconductors. Vol. 45 (7). P. $974-979$ (2011).

[5] T.Y. Peng, S.Y. Chen, L.C. Hsieh C.K. Lo, Y.W. Huang, W.C. Chien, Y.D. Yao. J. Appl. Phys. Vol. 99 (8). P. 08H710-08H712 (2006).

[6] W. Ou-Yang, M. Weis, D. Taguchi, X. Chen, T. Manaka, M. Iwamoto. J. Appl. Phys. Vol. 107 (12). P. 124506-124510
(2010).

[7] J. Wang, L. Wang, L. Wang, Z. Hao, Yi Luo, A. Dempewolf, M. M ller, F. Bertram, J rgen Christen. J. Appl. Phys. Vol. 112 (2). P. 023107-023112 (2012).

[8] K.K. Ong, K.L. Pey, P.S. Lee, A.T.S. Wee, X.C. Wang, Y.F. Chong, Appl. Phys. Lett. 89 (17), 172111-172114 (2006).

[9] H.T. Wang, L.S. Tan, E. F. Chor. J. Appl. Phys. 98 (9), 094901-094905 (2006).

[10] Yu.V. Bykov, A.G. Yeremeev, N.A. Zharova, I.V. Plotnikov, K.I. Rybakov, M.N. Drozdov, Yu.N. Drozdov, V.D. Skupov. Radiophysics and Quantum Electronics. Vol. 43 (3). P. 836-843 (2003).

[11] V.V. Kozlivsky. Modification of semiconductors by proton beams (Nauka, Sant-Peterburg, 2003, in Russian).

[12] E.L. Pankratov. Phys. Rev. B. Vol. 72 (7). P. 075201-075208 (2005).

[13] E.L. Pankratov. J. Appl. Phys. Vol. 103 (6). P. 064320-064330 (2008)

[14] E.L. Pankratov, E.A. Bulaeva. J. Comp. Theor. Nanoscience. Vol. 10 (4). P. 888-893 (2013).

[15] Z.Yu. Gotra, Technology of microelectronic devices (Radio and communication, Moscow, 1991, in Russian).

[16] V.I. Lachin, N.S. Savelov, Electronics (Phoenix, Rostov-na-Donu, 2001, in Russian).

[17] V.L. Vinetskiy, G.A. Kholodar', Radiative physics of semiconductors. ("Naukova Dumka", Kiev, 1979), (in Russian)

[18] Yu.D. Sokolov. Applied Mechanics. Vol.1 (1). P. 23-35 (1955).

[19] E.L. Pankratov. Eur. Phys. J. B. Vol. 57 (3). P. 251-256 (2007) 